\title{
VIOLÊNCIA NA GESTAÇÃO: UM ESTUDO DA PRODUÇÃO CIENTÍFICA DE 2000 A 2005
}

\author{
Violency during pregnancy: a study of the scientific \\ production from 2000 to 2005 \\ Violencia durante la gestación: un estudio de la producción \\ científica de 2000 a 2005
}

Ana Beatriz Campos Medina ${ }^{1}$

Lucia Helena Garcia Penna ${ }^{2}$

\section{RESUMO}

A violência à mulher durante a gestação e suas repercussões perinatais é um tema que abrange em sua totalidade situações comuns do nosso dia-a-dia, trazendo em particular aspectos que envolvem as relações humanas e o contexto sócio-cultural. 0 presente estudo pretende contribuir para uma reflexão a partir do conhecimento já produzido sobre violência na gravidez, fornecendo aos profissionais um panorama sobre o assunto, viabilizando o entendimento e a discussão da temática. É apresentada uma revisão bibliográfica da produção científica na América Latina e no Caribe sobre violência à gestante na área da saúde pública no período de 2000 a 2005. As maneiras de cuidar, ouvir, orientar, proporcionar confor to e alívio a essas mulheres, objetivando melhorar sua auto-estima e favorecer a compreensão de seus direitos, atentando para a redução dos agravos à saúde da díade mãe-filho, ainda são um desafio no atendimento realizado pelos profissionais de saúde.

Palavras-chave: Violência contra a Mulher. Saúde da Mulher

\begin{abstract}
Violence against women during pregnancy and the prenatal repercussions is a topic that includes or comprises totality common situations of our daily life, including particular aspects that involve human relations and the socio-cultural context.

The present study aims to contribute to the reflection (consideration) of the knowledge already produced about violence during pregnancy, providing the professionals with a broader view of this subject, making the understanding and the discussion about the theme possible. A bibliographical review of the scientific production in Latin America and the Caribbean about the violence against pregnant women in the public health area between the years of 2000 and 2005 is presented. The ways of caring for, listening to, counseling, giving comfort and relief to these women, having as an objective to improve their self-esteem and to favor the comprehension of their rights taking into consideration the reduction of the damages to the health of the binomial mother-son, is still a challenge in the assistance made by the health professionals.
\end{abstract}

Keywords: Pregnancy. Violence against women. Women's health

\section{Resumen}

La violencia a la mujer durante la gestación y sus repercusiones perinatales es un tema que abarca en su totalidad situaciones comunes de nuestro cotidiano, originando, en particular, aspectos que involucran las relaciones humanas y el contexto sociocultural. Esta investigación tiene el objetivo de contribuir para una reflexión del conocimiento producido hasta ahora sobre la violencia durante el embarazo, ofreciendo a los profesionales un panorama sobre el asunto, siendo posible el entendimiento y el análisis del tema Se presenta un repaso bibliográfico de la producción científica en América Latina y El Caribe sobre violencia a la embarazada en el área de la salud pública en el período de 2000 a 2005. Las formas de asistir, escuchar, dar consejos, proporcionar bienestar y alivio a estas mujeres con la intención de mejorar su autoestima y favorecer la comprensión de sus derechos considerando la disminución de los perjuicios a la salud del binomio madre-hijo, aún es un desafío en el atención y cuidado realizado por los profesionales de la salud.

Palabras-claves: Embarazo. Violencia contra la mujer. Salud de la mujer

${ }^{1}$ Especialista em Enfermagem Obstétrica, Mestranda do Programa de Pós-Graduação em Enfermagem da UERJ, Enfermeira da Casa de Parto David Capistrano Filho - SMS/RJ e da UTI Neonatal do Hospital Universitário Pedro Ernesto - UERJ - e-mail: beatriz-medina@terra.com.br ${ }^{2}$ Professora Adjunta do Departamento de Enfermagem Materno-infantil e do Programa de Mestrado em Enfermagem da UERJ 


\section{INTRODUÇÃO}

Segundo dados da Organização Mundial de Saúde', a violência é uma das principais causas de morte em todo 0 mundo para a população de 15 a 44 anos, constituindo um importante problema de saúde pública. Por esta razão, as situações de violência e seus agravantes vêm sendo amplamente discutidos e abordados nos mais diversos segmentos da sociedade moderna. Envolvem de forma abrangente os aspectos sociais, culturais, as diferenças de sexo e raças, as hegemonias e suas minorias, revelando no cotidiano a individualidade das relações pessoais e do contexto em que estão inseridas.

As diversas formas de violência que acometem os indivíduos no seu dia-a-dia repercutem de maneira significativa na sua saúde física e mental. Para minimizar seus conseqüentes agravos, é necessário um olhar criterioso e profundo voltado para a atenção à saúde com uma abordagem integral, individual e contextualizada.

Ao discutirmos as questões de saúde da mulher, identificamos a violência como fator de profunda relevância, e a violência doméstica é considerada uma das ameaças mais sérias, pois ocorre no seio da família, sendo perpetrada geralmente por um agressor de convívio íntimo com graus de parentesco familiar ou conjugal, em que a condição de mantenedor ou mesmo as relações de gênero favorecem um estado de superioridade em relação aos demais integrantes do núcleo familiar.

A violência, seja ela física, sexual, psicológica ou emocional, torna-se ainda mais séria quando a mulher se encontra grávida, pois traz conseqüências significativas para a saúde da díade mãe-filho, tais como baixo peso ao nascer, abortos, parto e nascimento prematuro e até mortes materna e fetal, conforme estudos revelados pela OMS ${ }^{1}$ no Informe Mundial sobre a Violência e a Saúde.

Os movimentos sociais enfatizavam uma visão mais ampla e integral à mulher, tendo importante significado social e constituindo-se em elemento fundamental de debates num momento histórico de democratização da sociedade brasileira. É a partir desses movimentos que começam a surgir incentivos públicos voltados para a prevenção e detecção de situações de violência relacionadas à mulher.

A atenção à saúde da mulher por meio de política pública tem, nas últimas décadas, incluído ações que contemplem as particularidades dos diferentes grupos populacionais e as condições sociais, econômicas, culturais e afetivas que interferem na condição de saúde da mulher. 0 Programa de Assistência Integral à Saúde da Mulher (PAISM) ${ }^{2}$ criado em 1984 marca uma ruptura conceitual com os princípios norteadores da política de saúde das mulheres e os critérios para a eleição de prioridades neste campo.

Apesar de ser considerado um marco na assistência à saúde integral da mulher, tendo por base a assistência ao pré-natal, parto e puerpério, a prevenção e controle do câncer cérvico- uterino e de mama e o planejamento familiar, verificamos que 0 PAISM apresenta algumas lacunas, como: a atenção ao climatério/menopausa, queixas ginecológicas, infertilidade e reprodução assistida, saúde da mulher na adolescência, doenças crônico-degenerativas e infecto-contagiosas e a inclusão da perspectiva de gênero e raça nas ações a serem desenvolvidas². Assim, não atende a toda necessidade da mulher nos seus diversos contextos sociais, sendo valorizados ainda, sobretudo, os aspectos clínicos e reprodutivos. No entanto, entendemos que houve com o PAISM uma tentativa de ruptura do modelo vigente à época que priorizava a atenção materno-infantil, com enfoque de assistência voltada para a criança.

Em 2004, a Política Nacional de Assistência Integral à Saúde da Mulher afirma que humanizar e qualificar a atenção em saúde é aprender a compartilhar saberes e reconhecer direitos, priorizando a organização de redes integradas de atenção às mulheres em situações de violência, assim como promover ações preventivas em relação à violência doméstica e sexual ${ }^{3}$.

É importante salientar que, embora as políticas de saúde estejam voltadas à prevenção e à atenção aos agravos à saúde da mulher, o cuidado implementado pelos profissionais ainda busca aprimoramento na tentativa da institucionalização de uma linguagem universal e uma abordagem multiprofissional que direcione as ações e atenda a necessidade da mulher.

0 cuidado humanizado à mulher no período perinatal surge como fator de importância na detecção e prevenção aos agravos gerados pelas situações de violência. Entendemos que esta maneira de cuidar vai além de ações terapêuticas, a subjetividade e a singularidade do eu e do outro é sentida e percebida, e há um momento de aproximação e de crescimento mútuo, pois os sujeitos trocam o que têm de mais íntimo, possibilitando assim o acontecer do verdadeiro cuidado ${ }^{4}$.

Torna-se de fundamental importância associar ao cuidado à mulher a família, avaliando seu contexto sócio-cultural, envolvendo a abordagem das relações entre os seus integrantes e o entendimento de que a existência de condições particulares individuais, familiares e coletivas aumenta o risco de ocorrência da violência no âmbito doméstico ${ }^{5}$.

Com um enfoque voltado para o emocional, o social e o cultural, o cuidado vislumbra o relacionamento entre a gestante e o pai do bebê como um ponto de importante avaliação, atentando para as expectativas na gestação, o desejo pelo bebê, o relacionamento com os diversos membros da família e a satisfação da mulher enquanto realização pessoal ${ }^{6}$.

Dessa maneira, entendemos a importância de avaliarmos a produção científica sobre o tema. Apesar de identificarmos um aumento significativo nos últimos anos, a publicação de trabalho na área da violência à mulher gestante ainda é considerada pequena. Existem vários fatores que dificultam a produção de registros sobre violência e gestação, entre eles a dificuldade dos profissionais da saúde em lidar com a situação, 
principalmente pela falta de uma linguagem comum em relação ao tema e pela falta de conceitos que reúnam o conhecimento em saúde ao de outras áreas ${ }^{7,8}$.

A violência vivenciada pela gestante ainda é de difícil abordagem nas relações pessoais e profissionais. As mulheres que vivenciam essas situações sentem-se coibidas em declarar as agressões de parceiros e familiares, e, ainda, alguns atos de violência não são reconhecidos por eles, nem por essas mulheres, e nem mesmo por profissionais de saúde.

No entanto, é através de produções e publicações de novos saberes acerca da violência na gestação que ela se caracteriza em sua existência, evidencia seus contornos e determina sua magnitude, exatamente pela capacidade de suscitar e legitimar o debate sobre sua ocorrência e importância.

0 presente estudo tem como finalidade contribuir para a reflexão sobre o conhecimento produzido acerca da violência contra a mulher gestante, fornecendo aos profissionais de saúde um panorama sobre o tema. Assim, o objetivo é analisar a produção científica na área de saúde pública, relativa à violência na gestação, nos últimos cinco anos.

\section{METODOLOGIA}

Elegemos os princípios da pesquisa bibliográfica, envolvendo as atividades básicas de identificação, compilação e fichamentos dos trabalhos. No primeiro momento de nossa pesquisa consultamos a Biblioteca Virtual de Saúde Pública (BVS-SP) através do site http://www.saudepublica.bvs.br, e a partir dela foi pesquisada a base de dados LILACS (Literatura Latino-Americana e do Caribe em Ciências da Saúde), que é parte da Biblioteca Virtual de Saúde (BVS) da Bireme, onde encontramos 72 produções bibliográficas utilizando as palavraschave: gravidez e violência e 30 produções com as palavraschave: violência e gestação. Desse total (102 produções), apenas 14 produções atenderam aos critérios escolhidos para alcançar os objetivos e desenvolver este estudo. Destacamos como critérios de inclusão: o período das publicações (2000 a 2005); produções que descrevessem explicitamente a relação da violência com a gestação e suas implicações no cuidado à mulher; a descrição do tema violência, gravidez e/ou gestação no resumo da base de dados (LILACS); característica de produção científica (artigos científicos, dissertações, teses ou monografias). Ressaltamos a exclusão de produções com temas correlatos (por exemplo, saúde reprodutiva) e manuais, cartilhas ou programas políticos e planejamentos estratégicos de assistência.

No segundo momento realizamos leitura das 14 produções científicas submetendo-as à seguinte classificação: ano e local de publicação; idéia principal/tendências conceituais; metodologia; classificação da produção científica. Em relação à característica metodológica dos estudos, observamos que quatro produções são reflexões teóricas ${ }^{14,15,18,22}$, quatro são pesquisas quantitativas ${ }^{11,13,17,21}$, uma caracteriza-se como estudo de caso clínico ${ }^{12}$ e cinco, como pesquisas qualitativas ${ }^{16,19,20,23,24}$.

No terceiro momento, realizou-se uma abordagem qualitativa dos dados utilizando-se a técnica de análise temática. Essa técnica "consiste em descobrir os núcleos de sentido que compõem a comunicação e cuja presença pode significar alguma coisa para o objetivo analítico escolhido". Além de se buscarem respostas para questões, pode-se caminhar na direção da "descoberta do que está por traz dos conteúdos manifestos, indo além das aparências do que está sendo analisado" 9 . A partir da análise temática realizada emergiram quatro categorias. As categorias, segundo Minayo ${ }^{10}$, são empregadas para se estabelecer classificações, neste sentido, trabalhar com elas significa agrupar elementos, idéias ou expressões em torno de um conceito capaz de abranger tudo isso.

\section{RESULTADOS E DISCUSSÃO}

Das 14 produções bibliográficas, nove são artigos de revistas ${ }^{11,12,13,14,15,16,17,18,19}$; quatro, teses de doutorado $20,21,22,23$ , e uma dissertação ${ }^{24}$. Verificamos que 13 delas são produtos de autores diretamente ligados às unidades de ensino superior $11,12,13,14,16,17,18,19,20,21,22,23,24$ e uma é ligada à instituição governamental de saúde (OPAS/OMS ${ }^{15}$ ).

Sabemos que as produções que envolvem a temática violência e gestação são escassâñ a relação da violência com a gestação, ${ }^{\prime \prime}$. Em nosso estudo, observamos que a produção apresentou um crescente a partir do ano de 2003. Identificamos que 10 produções são de origem nacional (oito da região sudeste ${ }^{14,16,18,20,21,22,23,24}$ e duas da região nordeste ${ }^{17,19}$ ) e quatro são produções internacionais ${ }^{11,12,13,15}$ (Chile, Venezuela, México e EUA). No que diz respeito à produção nacional, é possível justificá-la a partir do estabelecimento de políticas de saúde direcionadas à humanização da assistência à mulher, em especial à mulher no período gestacional ${ }^{25}$.

Tais políticas também são frutos de resultados alcançados por movimentos sociais de direitos humanos, em especial o feminista, e também de processos mais amplos presentes em toda a sociedade, como da consolidação democrática ${ }^{7}$. Internacionalmente, temos como marco a produção pela Organização Mundial de Saúde do Relatório Mundial sobre Violência e Saúde ${ }^{01}$ que tem sua importância por oferecer visibilidade ao tema, assim como subsidiar profissionais e pesquisadores sobre possíveis ações de prevenção da violência e promoção da saúde.

Analisando o conteúdo dos estudos aqui encontrados estabelecemos quatro categorias:

$1^{a}$ Categoria - A identificação da violência na gestação e suas repercussões perinatais

A partir da produção investigada, construímos esta categoria em que destacamos publicações que avaliam a construção e a 
aplicação da versão síntese de protocolos de atendimento para a identificação da violência na gestação e que buscam obter informações através de instrumentos de identificação de situações de violência na gestação e suas repercussões perinatais.

Dos trabalhos relacionados nesta categoria, dois avaliaram os instrumentos para o rastreamento dos casos de violência relacionados à mulher grávida ${ }^{18,22}$. A primeira produção analisada ${ }^{18}$ apresenta a equivalência semântica da versão em português de um instrumento originalmente em inglês - Abuse Assessment Screen (AAS), recomendando uma versão síntese de um protocolo de identificação da violência na gestação para uso habitual. Sendo avaliada a tradução em etapas com uma apreciação formal de equivalência e crítica, concluiu-se que é importante a inclusão de um momento adicional de interlocução do instrumento com membros da população-alvo, para que itens traduzidos possam mostrar outras versões de significados entre o original e a versão proposta.

A tese de doutoramento publicada em $2001^{22}$ avalia a confiabilidade e a validade de duas ferramentas diagnósticas - AAS e o Revised Conflict Tactics Scales - CTS2, que é empregado na constatação da violência entre casais. As diferenças existentes entre as definições, crenças e comportamentos relacionados ao tema impõem que a utilização desses instrumentos seja precedida de avaliações com a comparação de resultados e perfis epidemiológicos entre as diferentes localidades e culturas, sendo valiosa a investigação de equivalência transcultural.

Os outros artigos ${ }^{11,22}$ utilizam como base de avaliação o mesmo instrumento $A A S$, para estudar as conseqüências da violência na gestação sobre a saúde reprodutiva e neonatal. Nesta análise, observamos a importância da identificação e da intervenção nos casos de violência através do atendimento pré-natal, correlacionando-os aos fatores de risco associados e, identificamos as conseqüências da violência doméstica sobre a mulher grávida e seu bebê, com importantes índices de morbimortalidade perinatal.

$2^{\mathrm{a}}$ Categoria - A relação da violência e a gestação:

Esta categoria foi construída a partir das produções que apontavam a relação da violência diretamente com a gestação; identificamos artigos que valorizam a perspectiva da mulher gestante e da gestante adolescente.

Nos artigos analisados ${ }^{13,14}$, observamos a colocação da gravidez como um fator de risco ou um fator de proteção em situações de violência doméstica, comparando a prevalência e a gravidade relacionada às mulheres que vivenciam a violência antes e durante a gestação. Ao mesmo tempo, os conceitos de violência conjugal na gestação são revistos com uma abordagem na violência de gênero. É dado destaque à importância da identificação dos diversos cenários de risco e à intervenção eficaz pelos profissionais de saúde como passo fundamental para a prevenção e o tratamento da violência à mulher. 0 profissional de saúde encontra-se em posição privilegiada, pois, na maioria das vezes, independentemente da situação de violência que a mulher vivenciou, ou mesmo como ela se encontra, o serviço de saúde é o lugar que a mulher elege para buscar ajuda.

A tese de doutorado ${ }^{21}$ destaca-se pela avaliação da influência de fatores como a faixa etária dando enfoque à adolescência, à condição social da mulher, quem são os agressores e o local da agressão. Concentra-se na importância dos dados para subsidiar os programas de prevenção à violência intrafamiliar.

$3^{\mathrm{a}}$ Categoria - A relação entre a mulher e o profissional

$\mathrm{Na}$ análise encontramos produções que enfatizam a importância do profissional de saúde em promover um cuidado personalizado, que ajude a mulher a ter o seu bem-estar e a saúde, podendo agir e decidir cuidados de enfermagem que preservem, acomodem ou repadronizem o cuidado culturalmente conhecido e praticado por elas. Destacam a legitimidade dos profissionais de saúde para oferecer as modernas tecnologias, no entanto, consideram o saber empírico que as mulheres trazem sobre os processos de viver, adoecer, engravidar, parir, criar seus filhos e cuidar de sua família e sua comunidade ${ }^{19,20,23}$.

Evidenciam o aprofundamento das discussões na capacitação dos profissionais de saúde quanto às questões relacionadas às desigualdades de gênero e a reprodução de preconceitos não superados na nossa cultura ${ }^{20}$.

A importância dos profissionais de saúde em oferecer apoio e informação é discutida através da análise de situações observadas na unidade hospitalar, onde são atendidas gestantes em situações de violência, dando ênfase à necessidade de sensibilização destes profissionais e favorecendo a discussão do tema com as mulheres atendidas, o que facilita a elaboração de perguntas e o reconhecimento de lesões sugestivas de violência ${ }^{12}$.

Estas produções compreendem a necessidade da estruturação do processo comunicativo no ambiente hospitalar, com uma comunicação livre de bloqueios que valorize a clientela, demonstrando respeito às suas queixas, melhorando sua autoestima e incentivando-as a colaborar mais com a resolução dos seus problemas de saúde. Os profissionais de saúde não podem se considerar os únicos detentores do saber científico e técnico, mas estar preparados para a abordagem cultural, pois a complexidade que permeia as relações de gênero é relevante para que o cuidado possa facilitar na promoção do cuidar $^{23}$.

$4^{\mathrm{a}}$ Categoria - A relação da violência e a morbimortalidade materna:

Esta categoria emergiu das produções que destacam a relação existente entre a situação de violência na gestação e sua conseqüência sobre a morbimortalidade materna. Um dos achados importantes está na relação da ocorrência de situações de violência física conjugal com o ganho de peso gestacional|15,16,24. Destaca-se a necessidade de inovações na abordagem prática no atendimento pré-natal com o intuito de minimizar o possível déficit de ganho de peso durante a 
gestação, o que acarreta a ocorrência do aumento da morbimortalidade materna.

A preocupação com os índices epidemiológicos e as intervenções na saúde direcionam para a análise de um artigo ${ }^{15}$ que trabalha a reflexão teórica e social ampliada sobre o tema e ainda evidencia a importância de um conceito de morte por violência doméstica, preocupando-se com a elaboração de um plano estratégico com ações que envolvam a saúde da mulher e a igualdade de gênero.

A visão biomédica está presente na análise de atendimentos realizados à gestante com traumas abdominais, numa unidade hospitalar, correlacionados a situações de violência ${ }^{16}$. 0 artigo explicita a preocupação em incorporar ferramentas de avaliação obstétrica e de trauma no desenvolvimento de escore para estimativa de lesões que possam interferir na evolução e avaliação prognóstica materna e fetal.

Estas produções avaliam os fatores indicativos da evolução materna e fetal entre gestantes vítimas de situações de violência grave, analisam os casos de morte materna e suas relações com a violência doméstica e apresentam as dificuldades em identificar os indicadores de morbimortalidade materna associados à importância da prevenção e do cuidar.

\section{CONSIDERAÇÕES FINAIS}

As produções recentes sobre 0 assunto apontam possibilidades para o cuidar das mulheres que vivenciam violência, particularmente no período gestacional. Entretanto, na prática, ainda existem dificuldades na abordagem integral à mulher e sua família, assim como um entendimento limitado da equipe de saúde acerca das diversas situações contextuais (sócio-culturais) que podem estar diretamente relacionadas aos atuais índices de morbimortalidade perinatal, como, por exemplo, a ocorrência de situações de violência intrafamiliar.

Quanto à análise das propostas e recomendações relacionadas ao cuidar de mulheres que vivenciam a violência na gestação, observamos que as produções apontam para a importância da detecção da violência na gestação, e dessa forma apresentam instrumentos que buscam facilitar essa investigação, indicam a importância da sensibilização dos profissionais envolvidos na assistência à saúde, estimulam a investigação dos agressores, propõem a prevenção com ações eficientes e a estruturação do processo comunicativo entre mulher e profissional.

São notórias, nas produções, discussões direcionadas para a importância da atenção dos profissionais de saúde a essa população, principalmente destacando a proximidade com a clientela, enfatizando a necessidade de uma linguagem comum, a utilização de ferramentas de diagnóstico e a avaliação na identificação dos sinais e sintomas de violência e de intervenções eficazes, ancoradas na autonomia feminina e na garantia de seus direitos.
Consideramos a aplicação na prática de um instrumento de investigação de situações de violência à gestante um dos primeiros passos, mas entendemos ser necessário incorporar atenção às questões relacionadas à família, às condições sócioculturais e à história de vida da mulher, assim como às dificuldades institucionais, vivenciadas pela clientela e pelo profissional, durante a abordagem e o cuidar das mulheres que vivenciam violência.

Somam-se a esses fatos as recomendações aos profissionais de saúde, no sentido de perceberem de maneira mais próxima e livre de conceitos pré-concebidos, no que diz respeito à vivência do ciclo de violência, a dificuldade que a mulher tem em reconhecer e denunciar atitudes praticadas diariamente que são banalizadas com o passar do tempo.

Podemos enfatizar que os profissionais são os principais elos no atendimento e acompanhamento das situações de violência. Portanto, é de fundamental importância a abordagem da temática, incorporando o tema nos currículos escolares e nas unidades de saúde, com discussões multidisciplinares voltadas para a análise e acompanhamento dos casos atendidos, o contínuo incentivo às pesquisas e conseqüentes produções sobre 0 assunto, assim como o trabalho interdisciplinar com os profissionais envolvidos com o tema, objetivando a sensibilização e a atenção para a temática da violência na gravidez.

\section{REFERÊNCIAS}

1.Organização Panamericana de Saúde- OPS. Informe Mundial Sobre la Violência y la Salud. Washington (USA); 2002.

2.Ministério da Saúde (BR). Programa de Assistência à Saúde da Mulher: bases de ação programática. 1a ed. Brasília (DF); 1984.

3.Ministério da Saúde (BR). Política Nacional de Atenção Integral à Saúde da Mulher: princípios e diretrizes. $1^{\text {a }}$ ed. Brasilia (DF); 2004.

4.Celich KLS. Dimensões do processo de cuidar: a visão das enfermeiras. $1^{\text {a }}$ ed. Rio de Janeiro (RJ): EPUB; 2004.

5.Ministério da Saúde (BR). Violência intrafamiliar: orientações para prática em serviço. $1^{a}$ ed. Brasília (DF); 2001.

6.Maldonado MT. Psicologia da gravidez. Rio de Janeiro (RJ): Saraiva; 1996.

7.Penna LHG, Santos NC, Souza ER. A produção científica sobre violência doméstica na área da saúde pública. Rev Enferm UERJ 2004 ago; 12 (2): 192- 98.

8.Penna LHG. A temática da violência contra a mulher na formação da enfermeira [tese de doutorado]. Rio de Janeiro (RJ): Instituto Fernandes Figueiras/FIOCRUZ; 2005.

9.Bardin L. Análise de conteúdo. Lisboa (PO): Ed 70; 1977.

10.Minayo MCS, organizadora. Pesquisa social: teoria, método e criatividade. 15a ed. Rio de Janeiro (RJ): Vozes; 2000.

11.Arcos GE, Urac UM, Molina VI, Repossi FA, Ulloa VM. Impacto de la violencia doméstica sobre la salud reproductiva y neonatal. Rev Medica Chile 2001 dez; 129 (12):1413-24.

12.Benitez GG, Peña A, Peña D. Violência contra la mujer durante el embarazo: resumen de casos. Rev Obstet Ginecol, Venezuela 2004 dez; 64 (4): 211-13. 
13.Castro R, Ruiz A. Prevalencia y severidad de la violência contra mujeres embarazadas. Rev Saude Publica 2004 fev; 38 (1): 62-70.

14.Couto TM, Lopes RLM. Ser-mulher que vivenciou violência conjugal na gestação. Rev Enferm UERJ 2003 set/dez; 11(3): 296-99.

15.Espinoza H, Camacho AV. Muerte materna ocasionada por violência doméstica: componente critico e inadvertido de la mortalidad materna. Rev Panam Salud Publica 2005 fev; 17(2): 123-29.

16.Fraga GP, Mantovani M, Mesquita AC, Soares AB, Passini JR. Trauma abdominal em grávidas. Rev Bras Ginecol Obstet 2005 jun; 24 (9): 541-47.

17. Menezes TC, Amorim MMR, Santos LC, Faúndes A. Violência física doméstica e gestação: resultados de um inquérito no puerpério. Rev Bras Ginecol Obstet 2003 jun; 25(5): 30916.

18. Reichenheim ME, Moraes CL, Haselmann MH. Equivalência semântica da versão em português do instrumento Abuse Assessment Screen- AAS para rastrear a violência contra a mulher grávida. Rev Saude Publica 2000 dez; 34 (6): 610-16

19.Ximenes LB, Varela ZMV. As implicações de um novo evento gravídico na família de uma mulher multigesta. Acta Sci Health Sci 2004 out; 26 (2): 311-17.
20.Cavalcanti L F. Ações da assistência pré-natal voltadas para a prevenção da violência sexual: representações e práticas dos profissionais de saúde [tese de doutorado]. Rio de Janeiro (RJ): Instituto Fernandes Figueiras/FIOCRUZ; 2004.

21.Flores Sullca T. Violência como fator de risco para gravidez na adolescência. [tese de doutorado]. São Paulo (SP): Escola Paulista de Medicina/ USP; 2004.

22. Moraes CL. Aspectos metodológicos relacionados a um estudo sobre a violência familiar durante a gestação como fator de propensão da prematuridade do recém-nascido. [tese de doutorado]. Rio de Janeiro (RJ): Escola Nacional de Saúde Pública/FIOCRUZ; 2001.

23.Pereira WR. Poder, violência e dominação simbólicos em um serviço de saúde que atende mulheres em situação de gestação, parto e puerpério [tese de doutorado]. São Paulo (SP): Escola de Enfermagem de Ribeirão Preto/USP; 2000.

24.Amorim AR. Diferenciais de ganho de peso: o papel da violência física conjugal durante a gestação. [dissertação de mestrado]. Rio de Janeiro (RJ): Instituto de Medicina Social /UERJ; 2004.

25.Ministério da Saúde (BR). Programa Nacional de Humanização da Assistência Hospitalar. $1^{\mathrm{a}}$ ed. Brasília (DF); 2001. 